\title{
Dose-volume effects on brainstem dose tolerance in radiosurgery
}

\author{
Clinical article
}

\author{
Jinyu Xue, Ph.D., ${ }^{1}$ H. Warren Goldman, M.D., Ph.D., ${ }^{2}$ Jimm Grimm, Ph.D., ${ }^{3}$ \\ Tamara LaCouture, M.D., ${ }^{1}$ Yan Chen, Ph.D., ${ }^{1}$ Lesley Hughes, M.D., ${ }^{1}$ \\ and Ellen Yorke, Ph.D. ${ }^{4}$
}

Departments of ${ }^{\prime}$ Radiation Oncology and ${ }^{2}$ Neurological Surgery, Cooper University Hospital, Camden, New Jersey; ${ }^{3}$ Holy Redeemer Hospital, Meadowbrook, Pennsylvania; and ${ }^{4}$ Department of Medical Physics, Memorial Sloan-Kettering Cancer Center, New York, New York

Object. Dose-volume data concerning the brainstem in stereotactic radiosurgery (SRS) for trigeminal neuralgia (TN) were analyzed in relation to associated complications. The authors present their set of data and compare it with currently cited information on brainstem dose tolerance associated with conventional fractionated radiation therapy and hypofractionated radiation treatment of other diseases.

Methods. Stereotactic radiosurgery for TN delivers a much higher radiation dose to the brainstem in a single fraction than doses delivered by any other procedures. A literature survey of articles on radiosurgery for TN revealed no incidences of severe toxicity, unlike other high-dose procedures involving the brainstem. Published data on brainstem dose tolerance were investigated and compared with dose-volume data in TN radiosurgery. The authors also performed a biological modeling study of dose-volume data involving the brainstem in cases of TN treated with the Gamma Knife, CyberKnife, and linear accelerator-based systems.

Results. The brainstem may receive a maximum dose as high as 45 Gy during radiosurgery for TN. The major complication after TN radiosurgery is mild to moderate facial numbness, and few other severe toxic responses to radiation are observed. The biologically effective dose of $45 \mathrm{~Gy}$ in a single fraction is much higher than any brainstem dose tolerance currently cited in conventional fractionation or in single or hypofractionated radiation treatments. However, in TN radiosurgery, the dose falloff is so steep and the delivery so accurate that brainstem volumes of $0.1-0.5 \mathrm{~cm}^{3}$ or larger receive lower planned and delivered doses than those in other radiation-related procedures. Current models are suggestive, but an extensive analysis of detailed dose-volume clinical data is needed.

Conclusions. Patients whose TN is treated with radiosurgery are a valuable population in which to demonstrate the dose-volume effects of an extreme hypofractionated radiation treatment on the brainstem. The result of TN radiosurgery suggests that a very small volume of the brainstem can tolerate a drastically high dose without suffering a severe clinical injury. The authors believe that the steep dose gradient in TN radiosurgery plays a key role in the low toxicity experienced by the brainstem.

(http://thejns.org/doi/abs/10.3171/2012.7.GKS12962)

\section{KeY Words - brainstem - dose-volume relationship • dose tolerance • stereotactic radiosurgery - complication - trigeminal neuralgia}

\footnotetext{
Abbreviations used in this paper: $\mathrm{BED}=$ biologically effective dose; CISS = constructive interference in the steady-state sequence; $\mathrm{CTEP}=$ Cancer Therapy Evaluation Program; DVH = dose-volume histogram; GKS = Gamma Knife surgery; LINAC = linear accelerator; LQ = linear-quadratic; NTCP = normal tissue complication probability; QUANTEC = Quantitative Analysis of Normal Tissue Effects in the Clinic; REZ = root entry zone; SRS = stereotactic radiosurgery; $\mathrm{SRT}=$ stereotactic radiation therapy; $\mathrm{TN}=$ trigeminal neuralgia.
}

7 He brainstem is a critical structure of dosimetric concern for most intracranial radiation treatments. For conventional fractionated radiation therapy, the Radiation Therapy Oncology Group's Protocol 0539 introduced a brainstem dose tolerance limit of a maximum dose of $60 \mathrm{~Gy}$ delivered in 30 fractions to a volume greater than $0.03 \mathrm{~cm}^{3}$ for intermediate- and high-risk meningiomas. This protocol represents the dose constraint 
for a low-complication risk in patients anticipating long survival. The QUANTEC report ${ }^{17}$ concluded that for conventional fractionated 3D conformal radiotherapy, the risk of clinically apparent brainstem complications increases markedly at maximum doses greater than 64 Gy. Although some reviewed studies have reported serious complications (brainstem necrosis leading to death or disabling neuropathy), minor complications were also included ${ }^{17}$

For SRS and hypofractionated SRT, there are few published studies on brainstem toxicity in which both dosimetric and toxicity data are combined. The QUANTEC report, based on very limited data, cited maximum point doses below 12.5 Gy (SRS) as associated with less than $5 \%$ serious complications but noted that a low incidence of complication has also been reported for higher doses (15-20 Gy) in patient groups with poor prognoses for long-term survival. A 3\% calculated isocomplication at 14.2 Gy in a singleinstitution study of radiosurgery for a vestibular schwannoma was also cited in the report. ${ }^{18}$ There are currently no definitive criteria regarding more subtle dose-volume effects after SRS or SRT, and the data are insufficient to generate a unified dose-response curve covering the range from conventional fractionated radiation treatments through SRS. It is noteworthy that the literature that was reviewed for the QUANTEC report considered the brainstem as a uniform rather than multifunctional organ, and the accuracy with which the planned dose distribution was delivered was not considered.

Trigeminal neuralgia is a disorder of the fifth cranial nerve for which radiosurgery is considered an effective and safe treatment. ${ }^{5}$ A focused beam arrangement is directed to the trigeminal nerve where it is adjacent to the brainstem, that is, at the REZ, which is located at the anterolateral aspect of the pons. Treatment typically prescribes a maximum dose of 75-90 Gy in a single fraction. Dose planning is typically done such that an isodose line between $20 \%$ and $50 \%$ of the prescription touches the brainstem. Consequently, the brainstem receives a significantly higher dose than implied by the QUANTEC guidelines.

The most common complication associated with TN radiosurgery is facial numbness, which is considered an endpoint of mild "neuropathy." Since TN is a noncancerous condition, patients have normal overall survival times. Understanding the incidence of brainstem injury from TN radiosurgery dose distributions should be of interest to intracranial SRS and SRT clinics. Nevertheless, the QUANTEC publication did not include any TN data. In the present article, we review the incidence of complications related to radiosurgery for $\mathrm{TN}$ that have been published in the literature and present a dose-volume data set that may lead to a better understanding of the brainstem dose-volume tolerance limits associated with SRS.

\section{Methods}

There is ongoing debate as to whether complications after TN radiosurgery are correlated with the volume of brainstem or the length of the trigeminal nerve that is irradiated..$^{18,24}$ Since both the brainstem and nerve receive significant doses for effective treatment, it is possible to find a statistical correlation between the radiation dose and each of these anatomical structures. Radiation-induced brainstem injury can be graded by the common toxicity criteria of the CTEP. ${ }^{17}$ The most common complication associated with TN radiosurgery is facial numbness, which can be assessed by the Barrow Neurological Institute facial hypesthesia scale and scoring system. Table 1 offers a comparison of the facial numbness endpoints between 2 different grading systems.

In the case of TN radiosurgery, no CTEP Grade 4 or 5 toxicity has been reported. Most cases are mild to moderate, and even severe facial numbness is neither permanent nor requiring of treatment, and may be considered Grade 3 toxicity by the CTEP definition. To explore the possible dependence of complications on the dose-volume characteristics of brainstem irradiation during TN radiosurgery and to compare these dose-volume characteristics with the dose-volume characteristics of brainstem complications observed in the radiation treatment of other diseases, we reviewed the literature on $\mathrm{TN}$ radiosurgery with modern treatment technologies used in the last 10 years. Only a few studies have published brainstem dose-volume data. The reported complication rates, primarily facial numbness, have a wide range of variation, even for the same treatment modality. We selected studies showcasing complications observed in 3 treatment modalities: the Gamma Knife, CyberKnife, and LINAC-based systems.

All 3 methods rely on high-resolution (1-mm slice thickness) MR imaging to visualize the nerve and its relation to the brainstem. The Gamma Knife uses stereotactic head-frame immobilization and is considered the gold standard for radiosurgery of TN. Figure 1 is a screenshot of an axial view of a Gamma Knife dose plan for TN. High-resolution CISS MR imaging ${ }^{23}$ is used to guide the placement of a single 4-mm shot at the REZ of the trigeminal nerve. The CyberKnife treatment requires facial mask immobilization, and the radiation dose is delivered by the 6-MV beam of an X-band LINAC mounted on a robotic arm and guided by real-time imaging-assisted target tracking. The CyberKnife dose plan is prepared using a CT image fused with an MR image for delineation of the nerve and brainstem; the 5-mm cone and trigeminal node set, which has a shorter source-to-axis distance are used. ${ }^{13}$ The LINAC-based SRS is performed using either stereotactic head-frame immobilization or facial mask immobilization with imaging guidance. Typically, the target is localized via an MR image fused with highresolution CT. A spherical dose distribution is delivered by 5-7 noncoplanar arcs through a circular collimator (4 or $5 \mathrm{~mm}$ in diameter). ${ }^{22,24}$ For all 3 modalities, given their use of high-resolution and good-quality MR and CT images, uncertainty in the calculation of the dose distribution to the brainstem due to variability in brainstem delineation can be minimized.

Few studies that we reviewed provided detailed dosevolume-complication information such as individual brainstem DVHs along with complications. Therefore, we performed a retrospective analysis of all $32 \mathrm{TN}$ cases treated with the Gamma Knife Perfexion at Cooper University Hospital between September 2007 and Janu- 


\section{Brainstem dose tolerance}

TABLE 1: Criteria of complication endpoints*

\begin{tabular}{ll}
\hline \multicolumn{1}{c}{ CTEP Common Toxicity Criteria } & \multicolumn{1}{c}{ BNI Facial Hypesthesia Scale } \\
\hline Grade 1, mild or asymptomatic & Grade I, no facial numbness \\
Grade 2, moderate, not interfering w/ ADL & Grade II, mild facial numbness that is not bothersome \\
& Grade III, somewhat bothersome facial numbness \\
Grade 3, severe interference w/ ADL, possible intervention & Grade IV, very bothersome facial numbness \\
Grade 4, life-threatening or disabling; intervention indicated & NA \\
Grade 5, death & NA \\
\hline
\end{tabular}

* This table shows the common toxicity criteria of the Cancer Therapy Evaluation Program, which grades brainstem injury on the basis of symptoms, and the Barrow Neurological Institute facial hypesthesia scale and scoring system, which is used to assess facial numbness. Abbreviations: $\mathrm{ADL}=$ activities of daily living; $\mathrm{BNI}=$ Barrow Neurological Institute; $\mathrm{NA}=$ not applicable.

ary 2011. Data were extracted to explore the correlation between postradiosurgery complications and brainstem DVHs. The patients consisted of 25 women and 7 men with a median age of 74 years (range $41-90$ years). The placement of a single 4-mm open shot was such that the isocenter was at the distal part of the trigeminal nerve and the $50 \%$ isodose line just touched the brainstem surface. The prescribed dose was either 80 or $85 \mathrm{~Gy}$ at $100 \%$ (maximum dose). None of the patients had previously undergone surgery or radiation treatment for TN or the brain. The median patient follow-up time was 25 months (range 4-39 months) after GKS. In addition to the data that we collected, the Stanford CyberKnife group (courtesy of Dr. John Adler) provided us with the mean DVH data associated with outcomes reported in Reference 13. Data on LINAC-based radiosurgery were pulled from Reference 8. These data are summarized in Table 2.

There are apparent differences between the radiationinduced brainstem injuries reported for larger-field treatments using conventional fractionation and conventional delivery techniques, which were reviewed by the QUANTEC group, and the low rate of complications observed for the extremely hypofractionated radiosurgery of $\mathrm{TN}$

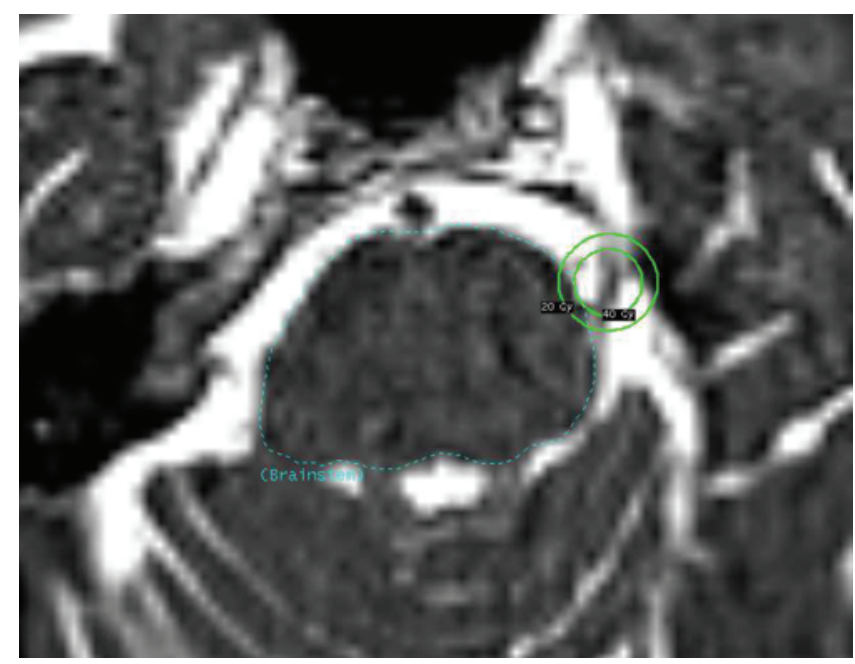

FIG. 1. Axial view of a high-resolution CISS MR image of the brain used for a GKS dose plan in the treatment of TN. Outlined are the brainstem and the radiation isodose lines for $40 \mathrm{~Gy}$ and $20 \mathrm{~Gy}$. treatments. We therefore attempted to model the normal tissue complication probability (NTCP) for the brainstem to see if parameters could be found that would cover both types of treatment observations, using both the Lyman ${ }^{14}$ and the Serial Architecture ${ }^{19}$ models. The LQ model was used to account for changes in biological effectiveness due to different doses per fraction. The models were applied to the data shown in Table 2. The equations for these models are given in the Appendix.

\section{Results}

Table 3 shows a comparison of complication data for TN radiosurgery across the various treatment modalities and dose schemes from publications that we reviewed. The most common complication was the development of facial numbness following the procedure. In the literature, the change in patients' facial sensation after TN radiosurgery is described by a mixture of terms such as "numbness," "paresthesias," "hypesthesia," and "trigeminal dysfunction." The reported rate of facial numbness in Table 3 includes the symptoms of both hypesthesia and paresthesia. As shown in Table 3, a wide range of facial numbness rates was reported from various studies, even when the same treatment modality was used. None of the publications we reviewed mentioned brainstem necrosis due to TN radiosurgery. Only 2 patients from Cooper University Hospital experienced moderate facial numbness. The complication rate in our patient group agrees with the results of GKS from some other institutions..$^{12,26}$

According to the LQ model, which is briefly described in the Appendix, the BED of a dose, D, delivered at a dose-per-fraction, d, is given by Equation A1. Regimens using the same BED are said to be isoeffective. Figure 2 plots the BED for the brainstem with $\alpha / \beta=3.0 \mathrm{~Gy}$ (the QUANTEC estimate for this tissue-specific parameter) for the doses per fraction to $0.03-\mathrm{cm}^{3}, 0.1-\mathrm{cm}^{3}$, and $1-\mathrm{cm}^{3}$ volumes in the Cooper TN radiosurgery series (see Table 2). The BEDs are $6 \mathrm{~Gy}\left(1 \mathrm{~cm}^{3}\right), 51 \mathrm{~Gy}\left(0.1 \mathrm{~cm}^{3}\right)$, and 131 Gy $\left(0.03 \mathrm{~cm}^{3}\right)$. The solid curves in Fig. 2 show the total doses given in multiple fractions that are isoeffective to these 3 TN data points. For example, 78 Gy delivered in 2-Gy fractions (39 treatments) is isoeffective to the single fraction dose delivered to $0.03 \mathrm{~cm}^{3}$ of the brainstem in the Cooper series. For a comparison with dose-volume guide- 
TABLE 2: Mean brainstem dose-volume data for TN radiosurgery from Cooper, Stanford, and UCLA*

\begin{tabular}{|c|c|c|c|c|c|}
\hline \multicolumn{2}{|c|}{ Gamma Knife (Cooper) } & \multicolumn{2}{|c|}{ CyberKnife (Stanford) } & \multicolumn{2}{|c|}{ LINAC (UCLA) } \\
\hline Dose (Gy) & Vol $\left(\mathrm{cm}^{3}\right)$ & Dose (Gy) & Vol $\left(\mathrm{cm}^{3}\right)$ & Dose (Gy) & $\operatorname{Vol}\left(\mathrm{cm}^{3}\right)$ \\
\hline 44.5 (max) & 0.0001 & 35.0 (max) & 0.0001 & 45.0 (max) & 0.008 \\
\hline 37.9 & 0.001 & 25.0 & 0.01 & 27.0 & 0.047 \\
\hline 18.4 & 0.03 & 18.0 & 0.03 & 18.0 & 0.14 \\
\hline 11.0 & 0.1 & 13.0 & 0.1 & & \\
\hline 3.0 & 1.0 & 3.0 & 1.0 & & \\
\hline
\end{tabular}

lines obtained from published analyses of other types of radiotherapy, the dose constraints cited in the QUANTEC report and the 1-, 3-, and 5-fraction guidelines for $1 \mathrm{~cm}^{3}$ of the brainstem published in a recent overview of hypofractionated radiation treatment ${ }^{1,25}$ are also shown in the graph.

Both a single fraction of 37.9 Gy (the TN $1-\mathrm{mm}^{3}$ dose-volume point) and a single fraction of $44.5 \mathrm{~Gy}$ (the TN $0.1-\mathrm{mm}^{3}$ dose-volume point) are isoeffective to more than 300 Gy in 2-Gy fractions. Although the LQ model is widely used, it likely overestimates cell killing at the higher doses per fraction used for TN treatment. ${ }^{3,10}$ Figure 2 illustrates the discrepancy between published brainstem dose-volume constraints and the LQ model's extrapolations from the TN dose-volume data. Therefore, an underlying question for Fig. 2 is the applicability of the LQ model to the $\mathrm{TN}$-ablative dose regimen.

Figure 3 shows a comparison of the brainstem dosevolume points from TN radiosurgery and the 1-, 3-, and 5 -fraction brainstem dose tolerance guidelines given in the report of the American Association of Physicists in Medicine Task Group 101. ${ }^{1}$ The TN doses to volumes of 1 $\mathrm{mm}^{3}$ or less are notably higher than those guidelines suggest, although doses to larger volumes fall rapidly below the $0.5-\mathrm{cm}^{3}$ tolerance values. This comparison indicates a small volume effect where an extremely high dose is tolerable, if the rest of the brainstem is accurately spared by much lower delivered doses.

Several models aim to predict the probability of a complication associated with a given dose distribution. For conventional fractionation, QUANTEC suggested Lyman model parameters of $\mathrm{TD}_{50}=72 \mathrm{~Gy}, \mathrm{n}=0.16$, and $\mathrm{m}=0.14$; or $\mathrm{TD}_{50}=65 \mathrm{~Gy}, \mathrm{n}=0.16$, and $\mathrm{m}=0.1$. For the Serial Architecture model, the whole-organ NTCP parameters $\left[\mathrm{D}_{50}\right.$ and $\mathrm{k}$ for $\mathrm{P}(\mathrm{D})$ in Equation A3] that match the conventional fractionation Lyman model with $\mathrm{TD}_{50}=72$ Gy and $\mathrm{m}=$ 0.14 are $\mathrm{D}_{50}=72 \mathrm{~Gy}$ and $\mathrm{k}=11$, whereas for $\mathrm{TD}_{50}=65 \mathrm{~Gy}$ and $\mathrm{m}=0.1, \mathrm{P}(\mathrm{D})$ matches well if $\mathrm{D}_{50}=65 \mathrm{~Gy}$ and $\mathrm{k}=16$. (See Appendix for further definitions.) We calculated the predicted NTCP for both models for the $3 \mathrm{TN}$ data sets. For these calculations, we converted the single-fraction doses to 2-Gy equivalents using the LQ model with $\alpha / \beta$ $=3 \mathrm{~Gy}$. We estimated the brainstem volume to be $40 \mathrm{~cm}^{3}$ and assumed that $39 \mathrm{~cm}^{3}$ received a zero dose while the

TABLE 3: Reported complications associated with TN radiosurgery from selected studies*

\begin{tabular}{|c|c|c|c|c|c|c|}
\hline $\begin{array}{l}\text { Treatment } \\
\text { Modality }\end{array}$ & $\begin{array}{c}\text { No. of } \\
\text { Patients }\end{array}$ & $\begin{array}{l}\text { Max Dose } \\
\text { (Gy) }\end{array}$ & FU (mos) & $\begin{array}{c}\text { Facial } \\
\text { Numbness (\%) }\end{array}$ & Other Complication & Institution \\
\hline Gamma Knife & 32 & $80-85$ & $4-39$ & 6 & no & Cooper \\
\hline Gamma Knife & 365 & 80 & 28 (median) & $\begin{array}{c}6.5 \text { (first) } \\
26 \text { (repeat) }\end{array}$ & no & Saint Elisabeth ${ }^{26}$ \\
\hline Gamma Knife & 100 & $70-90$ & $>12$ & 10 & no & Marseille ${ }^{21}$ \\
\hline Gamma Knife & 50 & $60-90$ & $11-36$ & 6 & no & multiinstitutional $^{12}$ \\
\hline Gamma Knife & 65 & 90 & 6 & 46 & no & Erasme $^{15}$ \\
\hline LINAC & 179 & $70-90$ & $>5$ & 50 & eye irritation/dryness & UCLA $^{24}$ \\
\hline LINAC & 28 & 80 & $1-40$ & 11 & neurotrophic keratopathy & Wisconsin ${ }^{22}$ \\
\hline CyberKnife & 41 & $71-86$ & $>6$ & 51 & $\begin{array}{l}\text { anesthesia dolorosa } \\
\text { decreased corneal reflex } \\
\text { ipsilateral masticator weakness } \\
\text { trismus }\end{array}$ & Stanford ${ }^{13}$ \\
\hline CyberKnife & 95 & $70-85.4$ & 24 (mean) & 47 & dysesthesia & multicenter ${ }^{27}$ \\
\hline
\end{tabular}

* FU = follow-up. 


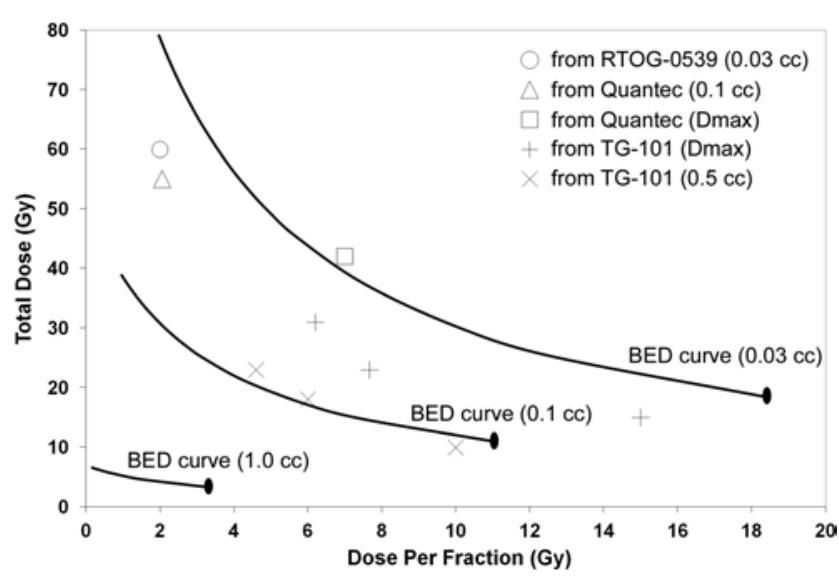

FIG. 2. Graph showing a comparison of published brainstem dosevolume constraints for different fractionation schedules with the 0.03 $\mathrm{cm}^{3}, 0.1-\mathrm{cm}^{3}$, and $1.0-\mathrm{cm}^{3}$ Cooper points from the mean brainstem DVH for single-fraction GKS for TN (ellipses). Multifraction total doses with the same BED as the TN points ( $\alpha / \beta=3 \mathrm{~Gy})$, shown as solid curves, are iso-BED. Dmax = maximum dose; RTOG-0539 = Radiation Therapy Oncology Group's Protocol 0539; TG-101 = American Association of Physicists in Medicine Task Group 101.

remaining $1 \mathrm{~cm}^{3}$ received the dose described in Table 2 . The calculated complication rates are summarized in Table 4 together with the observed outcomes.

\section{Discussion}

The only significant complication associated with radiation treatment of $\mathrm{TN}$ is facial numbness. The symptom of either paresthesias or hypesthesia is considered a mild "neuropathy" endpoint. Most numbness reported is mild or "moderate, not interfering with activities of daily living," that is, Grade 1 or 2 according to the CTEP criteria. Some have argued that those complications are not caused solely by radiation toxicity to the brainstem. For example, according to some publications the authors observed a statistical correlation between facial numbness and the length of the trigeminal nerve that was irradiated. ${ }^{6,13}$ Others have found that the dose to the brainstem is a significant factor for both pain relief and radiosurgeryinduced complications, including facial numbness, dry eye, dysgeusia, and so forth. ${ }^{9,16,24}$

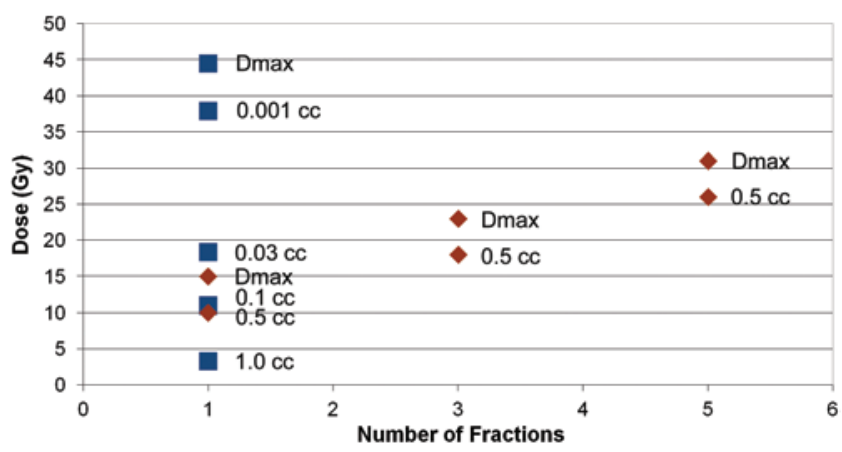

FIG. 3. Graph showing brainstem dose-volume values for TN radiosurgery from Cooper University Hospital (blue squares) compared with the brainstem dose tolerance values found by the American Association of Physicists in Medicine Task Group 101 (TG 101) (brown diamonds).
There is no consensus about the optimal radiation dose and targeting location, ${ }^{5}$ although targeting the distal nerve would reduce the dose to the brainstem. There is ongoing debate about the extent of nerve and brainstem to be irradiated. It would be interesting to carry out an analysis using DVH data for both the brainstem and the trigeminal nerve for a large patient group. Such a study would help us understand the correlation of pain control and complication with a dose to both anatomical structures and may also help determine the optimal dosing and targeting of TN radiosurgery. It is notable that no toxicity of CTEP Grades 4 and higher has been reported in treatments in which a small volume of brainstem in the vicinity of the REZ receives a dose of more than $40 \mathrm{~Gy}$ in a single fraction. No brainstem necrosis is reported to be associated with $\mathrm{TN}$ treatment in the literature that we reviewed, although this endpoint is clearly reported for conventional fractionated radiation treatments, in which DVHs typically show several cubic centimeters in the "high-dose" region. Nerve necrosis was observed at a dose of 100 Gy when GKS was used in an animal study. ${ }^{11}$

Meeks et al. ${ }^{18}$ used brainstem dose-volume data to estimate the rate of facial or trigeminal neuropathy from radiosurgery in the treatment of vestibular schwannomas. They found that the cranial neuropathies associated with radiosurgery for this lesion are a result of the dose received by the brainstem. ${ }^{18}$ A statistical correlation was also observed by Foote and colleagues ${ }^{7}$ between the dose to the brainstem and the probability of complication. Gorgulho and coauthors ${ }^{8}$ evaluated imaging changes on follow-up MR images after LINAC-based SRS was performed to treat TN. Their study showed that the gadolinium-induced enhancement of the brainstem at the REZ on MR images has both a significant correlation with pain relief $(p=0.0087)$ and a positive correlation with the incidence of numbness $(p=0.02)$. However, trigeminal nerve enhancement has no correlation with pain control after SRS $(p=0.22)$. In other words, better pain relief and a higher incidence of numbness are correlated to a higher radiation dose delivered to the REZ at the brainstem.

Patients with TN who are treated with radiosurgery are a valuable population in which to study dose-volume effects on brainstem-related complications at extreme hypofractionation. These patients are a long-lived population with reliable follow-up data. The brainstem suffers neither deformation nor internal motion during the course of image acquisition, dose planning, and delivery. The factor that determines the difference in calculated and delivered dose distribution is the accuracy of delivery. Stereotactic radiosurgery is supposed to have a much better delivery accuracy than conventional radiation therapy. Nevertheless, no publication is available that offers a comparison of the clinical accuracy of radiosurgery among the three SRS technologies discussed here, including an explicit study of mechanical, imaging, dosimetric, and patient immobilization accuracy. The delivery accuracy of GKS was evaluated in one publication and found to have a $0.91 \mathrm{~mm}$ targeting deviation and an $11 \%$ mean dose deviation. ${ }^{15}$ More studies of this sort are needed to unravel the dependence of observed brainstem complications and other outcomes on the delivered radiation dose. 
TABLE 4: Reported posttreatment complications from Cooper, Stanford, and UCLA compared with the NTCP calculated using the Serial Architecture and Lyman models*

\begin{tabular}{|c|c|c|c|}
\hline Characteristic & GK (Cooper) & CK (Stanford) & LINAC (UCLA) \\
\hline & \multicolumn{3}{|c|}{ Reported Complication Rate } \\
\hline total complications & $6 \%$ & $63 \%$ & $69 \%$ \\
\hline total facial numbness & $6 \%$ & $51 \%$ & $50 \%$ \\
\hline \multirow[t]{2}{*}{ moderate/severe numbness } & $6 \%$ & $15 \%$ & $14 \%$ \\
\hline & \multicolumn{3}{|c|}{ Calculated NTCP } \\
\hline $\begin{array}{l}\text { Serial Architecture } \\
\qquad D_{50}=72 \mathrm{~Gy}, \mathrm{k}=11\end{array}$ & $6 \%$ & $17 \%$ & $51 \%$ \\
\hline $\begin{array}{l}\text { Serial Architecture } \\
\qquad D_{50}=65 \mathrm{~Gy}, \mathrm{k}=16\end{array}$ & $11 \%$ & $29 \%$ & $79 \%$ \\
\hline $\begin{array}{l}\text { Lyman } \\
\qquad \mathrm{TD}_{50}=72 \mathrm{~Gy}, \mathrm{~m}=0.14, \mathrm{n}=0.16\end{array}$ & $16 \%$ & $11 \%$ & $100 \%$ \\
\hline $\begin{array}{l}\text { Lyman } \\
\qquad \mathrm{TD}_{50}=65 \mathrm{~Gy}, \mathrm{~m}=0.1, \mathrm{n}=0.16\end{array}$ & $32 \%$ & $21 \%$ & $100 \%$ \\
\hline
\end{tabular}

Modeling of brainstem complications is subject to other major uncertainties including the scarcity of dosevolume data and the questionable validity of the LQ model for very high dose fractions. Nonetheless, we attempted to use the limited data available as well as parameters that were consistent with those proposed by QUANTEC to see if either the frequently used Lyman model or the Serial Architecture model could deal with the spectrum of observed rates of brainstem complications for the dose distributions of conventional fractionated "largevolume" treatments and for TN treatment, in which very tiny volumes are irradiated to extremely high doses. The Serial Architecture predictions are more in line with the observations than those of the Lyman model, especially for LINAC-based TN treatments, in which only 3 dosevolume data points were available. To go beyond this limited study, it is necessary to acquire detailed dose-volume-outcome data in both conventional and radiosurgical regimens for all 3 treatment modalities and subject these and other models to more complete testing.

The results of radiosurgery in the treatment of TN suggest that a very small volume of brainstem can tolerate an extremely high dose without producing a severe clinical injury. It is interesting that animal studies of irradiation of the rat spinal cord also showed a steep increase in the tolerance dose for a $50 \%$ complication $\left(\mathrm{TD}_{50}\right)$ when decreasing the length of the cord from $8 \mathrm{~mm}\left(\mathrm{TD}_{50}=24.9\right.$ Gy) to $2 \mathrm{~mm}\left(\mathrm{TD}_{50}=87.8 \mathrm{~Gy}\right) .^{2}$ In addition, in a recent publication the authors reported that it is possible to safely irradiate extremely small volumes of the human spinal cord to median doses of 20 Gy in a single fraction using the CyberKnife system. ${ }^{4}$ Both brainstem and spinal cord are similar tissue, believed to have a serial organ structure. Those observations are somewhat contradictory to the common clinical assumption that delivery of a dose higher than a maximum threshold to an extremely small volume of a serial organ leads to severe complications. ${ }^{20}$ Of note, in all the scenarios mentioned here, the localiza- tion of critical structures during planning and delivery are subject to much tighter localization tolerances than is typical of conventional fractionated treatment.

Radiosurgery for TN has a much faster dose falloff than conventional radiation therapy. Such a steep dose gradient can be the reason why the brainstem can tolerate an extremely high dose delivered to a small volume. It is also possible that the small volume effect observed in TN treatment is related to the region of the brainstem that is irradiated. Perhaps the high dose reaches a region that, if damaged, has only minor clinical consequence. A study of radiation sensitivity of brainstem neuroanatomical details is beyond the scope of this manuscript, but this topic should be explored in the future as information becomes available.

\section{Conclusions}

Our analysis demonstrates that radiation-related brainstem toxicity in SRS for TN has a novel type of dose-volume effect. Numerous clinical reports show that there is very little severe radiation toxicity when a small volume of brainstem receives an extremely high dose of radiation with delivery methods that accurately localize the brainstem and maintain a steep dose gradient so that the rest of the organ is spared from high doses. The data from this patient group raise questions about our current understanding of the dose tolerance of the brainstem and the radiobiology associated with hypofractionated SRS and SRT. The risk of radiation toxicity must be considered as a dose-volume effect, rather than simply evaluated on the basis of a single point dose. Furthermore, within the brainstem, dose tolerance is possibly dependent on the region that is irradiated, and the use of one dose constraint for the entire brainstem may not be optimal. It is important to acquire dose-volume-outcome data for a better understanding of the brainstem response to radiation in both conventional and radiosurgery regimens. 


\section{Appendix}

\section{Linear-Quadratic Model}

The biological effect on an organ of a total dose, D, depends on the total dose, the dose per fraction, $d$, and the organ. Many results of in vitro and animal experiments and clinical outcomes are consistent with the LQ model, in which the BED is given by

$$
\mathrm{BED}=\mathrm{D}(1+\mathrm{d} /(\alpha / \beta))
$$

The parameter $\alpha / \beta$ must be experimentally determined for each tissue of interest.

Two fractionation schedules, $\left(\mathrm{D}_{1}, \mathrm{~d}_{1}\right)$ and $\left(\mathrm{D}_{2}, \mathrm{~d}_{2}\right)$ are said to be isoeffective (as in Fig. 2) if they have the same BED; that is, if

$$
\mathrm{D}_{1}\left(1+\mathrm{d}_{1} /(\alpha / \beta)\right)=\mathrm{D}_{2}\left(1+\mathrm{d}_{2} /(\alpha / \beta)\right)
$$

[Eq. A1a]

\section{Lyman Model}

The Lyman model has 3 parameters: $\mathrm{TD}_{50}$, the dose at which there is a $50 \%$ risk of complication for uniform irradiation of the entire organ; $n$, the "volume effect" parameter; and $m$, the responsesteepness parameter. Given a DVH in which volume fraction $v_{i}$ receives dose $D_{i}$, the effective dose $D_{\text {eff }}$ is calculated as

$$
D_{\text {eff }}=\left(\sum v_{\mathrm{i}}\left(D_{\mathrm{i}}\right)^{1 / \mathrm{n}}\right)^{\mathrm{n}}
$$

The normal tissue complication probability is given by

$$
\mathrm{NTCP}=100 \times \frac{1}{\sqrt{2 \pi}} \int_{-\infty}^{\left(D_{e f f}-T D_{50}\right) /\left(m T D_{50}\right)} e^{-t^{2} / 2} d t
$$

\section{Serial Architecture Model}

The organ is assumed to be made up of "functional subunits" such that complication results if one or more functional subunits are killed by radiation. In Reference 19, Equation A2 is derived for the NTCP resulting from a general dose distribution in which volume fraction $\mathrm{v}_{\mathrm{i}}$ receives dose $\mathrm{D}_{\mathrm{i}}$ in terms of $\mathrm{P}(\mathrm{D})$, the NTCP for uniform whole organ irradiation.

$$
\mathrm{NTCP}=100 \times\left(1-\Pi_{\mathrm{i}}\left(1-\mathrm{P}\left(\mathrm{D}_{\mathrm{i}}\right)\right)^{\mathrm{v}_{\mathrm{i}}}\right)
$$

For both models, the LQ theory can be used to convert each dose to the BED at the standard fractionation (for example, $2 \mathrm{~Gy} /$ fraction).

\section{Disclosure}

The authors report no conflict of interest concerning the materials or methods used in this study or the findings specified in this paper.

Author contributions to the study and manuscript preparation include the following. Conception and design: Xue. Acquisition of data: Xue. Analysis and interpretation of data: Xue, Grimm, Yorke. Drafting the article: Xue. Critically revising the article: all authors. Reviewed submitted version of manuscript: all authors. Approved the final version of the manuscript on behalf of all authors: Xue. Statistical analysis: Xue, Yorke. Study supervision: Goldman.

\section{References}

1. Benedict SH, Yenice KM, Followill D, Galvin JM, Hinson W, Kavanagh B, et al: Stereotactic body radiation therapy: the report of AAPM Task Group 101. Med Phys 37:4078-4101, 2010 (Erratum in Med Phys 39:563, 2012)

2. Bijl HP, van Luijk P, Coppes RP, Schippers JM, Konings AW, van der Kogel AJ: Dose-volume effects in the rat cervical spinal cord after proton irradiation. Int J Radiat Oncol Biol Phys 52:205-211, 2002

3. Chang BK, Timmerman RD: Stereotactic body radiation ther- apy: a comprehensive review. Am J Clin Oncol 30:637-644, 2007

4. Daly ME, Choi CY, Gibbs IC, Adler JR Jr, Chang SD, Lieberson RE, et al: Tolerance of the spinal cord to stereotactic radiosurgery: insights from hemangioblastomas. Int J Radiat Oncol Biol Phys 80:213-220, 2011

5. Elaimy AL, Hanson PW, Lamoreaux WT, Mackay AR, Demakas JJ, Fairbanks RK, et al: Clinical outcomes of gamma knife radiosurgery in the treatment of patients with trigeminal neuralgia. Int J Otolaryngol [epub ahead of print], 2012

6. Flickinger JC, Pollock BE, Kondziolka D, Phuong LK, Foote RL, Stafford SL, et al: Does increased nerve length within the treatment volume improve trigeminal neuralgia radiosurgery? A prospective double-blind, randomized study. Int J Radiat Oncol Biol Phys 51:449-454, 2001

7. Foote KD, Friedman WA, Buatti JM, Meeks SL, Bova FJ, Kubilis PS: Analysis of risk factors associated with radiosurgery for vestibular schwannoma. J Neurosurg 95:440-449, 2001

8. Gorgulho A, De Salles AA, McArthur D, Agazaryan N, Medin P, Solberg T, et al: Brainstem and trigeminal nerve changes after radiosurgery for trigeminal pain. Surg Neurol 66:127-135, 2006

9. Goss BW, Frighetto L, DeSalles AA, Smith Z, Solberg T, Selch M: Linear accelerator radiosurgery using 90 gray for essential trigeminal neuralgia: results and dose volume histogram analysis. Neurosurgery 53:823-830, 2003

10. Kirkpatrick JP, Meyer JJ, Marks LB: The linear-quadratic model is inappropriate to model high dose per fraction effects in radiosurgery. Semin Radiat Oncol 18:240-243, 2008

11. Kondziolka D, Lacomis D, Niranjan A, Mori Y, Maesawa S, Fellows W, et al: Histological effects of trigeminal nerve radiosurgery in a primate model: implications for trigeminal neuralgia radiosurgery. Neurosurgery 46:971-977, 2000

12. Kondziolka D, Lunsford LD, Flickinger JC, Young RF, Vermeulen S, Duma CM, et al: Stereotactic radiosurgery for trigeminal neuralgia: a multiinstitutional study using the gamma unit. J Neurosurg 84:940-945, 1996

13. Lim M, Villavicencio AT, Burneikiene S, Chang SD, Romanelli $\mathrm{P}, \mathrm{McNeely} \mathrm{L}$, et al: CyberKnife radiosurgery for idiopathic trigeminal neuralgia. Neurosurg Focus 18(5):E9, 2005

14. Marks LB, Yorke ED, Jackson A, Ten Haken RK, Constine LS, Eisbruch A, et al: Use of normal tissue complication probability models in the clinic. Int J Radiat Oncol Biol Phys 76 (3 Suppl):S10-S19, 2010

15. Massager N, Abeloos L, Devriendt D, Op de Beeck M, Levivier M: Clinical evaluation of targeting accuracy of gamma knife radiosurgery in trigeminal neuralgia. Int J Radiat Oncol Biol Phys 69:1514-1520, 2007

16. Massager N, Lorenzoni J, Devriendt D, Desmedt F, Brotchi J, Levivier M: Gamma knife surgery for idiopathic trigeminal neuralgia performed using a far-anterior cisternal target and a high dose of radiation. J Neurosurg 100:597-605, 2004

17. Mayo C, Yorke ED, Merchant TE: Radiation associated brainstem injury. Int J Radiat Oncol Biol Phys 76 (3 Suppl):S36S41, 2010

18. Meeks SL, Buatti JM, Foote KD, Friedman WA, Bova FJ: Calculation of cranial nerve complication probability for acoustic neuroma radiosurgery. Int J Radiat Oncol Biol Phys 47:597602,2000

19. Niemierko A, Goitein M: Calculation of normal tissue complication probability and dose-volume histogram reduction schemes for tissues with a critical element architecture. Radiother Oncol 20:166-176, 1991

20. Papiez L, Timmerman R: Hypofractionation in radiation therapy and its impact. Med Phys 35:112-118, 2008

21. Régis J, Metellus P, Hayashi M, Roussel P, Donnet A, BilleTurc F: Prospective controlled trial of gamma knife surgery for essential trigeminal neuralgia. J Neurosurg 104:913-924, 2006 
22. Richards GM, Bradley KA, Tomé WA, Bentzen SM, Resnick DK, Mehta MP: Linear accelerator radiosurgery for trigeminal neuralgia. Neurosurgery 57:1193-1200, 2005

23. Sheth S, Branstetter BF IV, Escott EJ: Appearance of normal cranial nerves on steady-state free precession MR images. Radiographics 29:1045-1055, 2009

24. Smith ZA, Gorgulho AA, Bezrukiy N, McArthur D, Agazaryan N, Selch MT, et al: Dedicated linear accelerator radiosurgery for trigeminal neuralgia: a single-center experience in 179 patients with varied dose prescriptions and treatment plans. Int J Radiat Oncol Biol Phys 81:225-231, 2011

25. Timmerman RD: An overview of hypofractionation and introduction to this issue of seminars in radiation oncology. Semin Radiat Oncol 18:215-222, 2008

26. Verheul JB, Hanssens PE, Lie ST, Leenstra S, Piersma H, Beute GN: Gamma Knife surgery for trigeminal neuralgia: a review of 450 consecutive cases. Clinical article. J Neurosurg 113 Suppl:160-167, 2010

27. Villavicencio AT, Lim M, Burneikiene S, Romanelli P, Adler JR, McNeely L, et al: Cyberknife radiosurgery for trigeminal neuralgia treatment: a preliminary multicenter experience. Neurosurgery 62:647-655, 2008

Manuscript submitted May 15, 2012.

Accepted July 24, 2012.

Please include this information when citing this paper: DOI: 10.3171/2012.7.GKS12962.

Address correspondence to: Jinyu Xue, Ph.D., Department of Radiation Oncology, Cooper University Hospital, One Cooper Plaza, Camden, New Jersey 08103. email: xue-jinyu@ cooperhealth. edu. 